\title{
Microbiology of Neonatal Gram-Negative Sepsis in A Level III Neonatal Intensive Care Unit (NICU). A Single Center Experience
}

\author{
Mustafa AlAbdullatif ${ }^{1 *}$, Hassib Narchi ${ }^{2}$, Nusrat Khan ${ }^{1}$, Aiman Rahmani ${ }^{1}$, Tasnim Alkhatib ${ }^{1}$, Omar Abu- \\ Sa'da ${ }^{1}$ and Mohammad Khassawneh ${ }^{1}$
}

${ }^{1}$ Tawam Hospital, Neonatology division, United Arab Emirates

${ }^{2}$ College of Medicine and Health Sciences, United Arab Emirates

*Corresponding author: Mustafa AlAbdullatif, Neonatology fellow, Department of Pediatrics, Tawam Hospital, Al Ain, United Arab Emirates.

Received Date: September 11, 2019

Published Date: September 17, 2019

\section{Abstract}

Background: Sepsis is still a leading cause of neonatal morbidity and mortality especially when caused by Gram-negative bacteria. The causative organisms and their susceptibility to antibiotics vary among units. Empiric antibiotic therapy is based on the likely pathogens and their susceptibility pattern in a NICU. This study aims to identify, in a cohort of neonates diagnosed with Gram-negative sepsis, the bacteriological profile and the antibiotic susceptibilities as well as to evaluate the appropriateness of the empirical antibiotic coverage.

Material and methods: In this retrospective observational study, all Gram-negative pathogens isolated in the blood culture of neonates admitted to the neonatal unit in a tertiary referral hospital between January 2011and December 2015 were analyzed. Demographic data, causative organisms, antibiotic susceptibility, empiric therapy and outcomes were collected and analyzed.

Results: Of the 2732 neonates admitted to NICU, 80 infants (2.9\%) had a blood culture-proven sepsis with a Gram-negative pathogen. Klebsiella pneumonia was the commonest causative organism. Sensitivity to gentamicin and meropenem were $95 \%$ and $99 \%$ respectively. Mortality, necrotizing enterocolitis, periventricular leukomalacia, bronchopulmonary dysplasia, intraventricular hemorrhage, retinopathy of prematurity was more prevalent in affected infants.

Conclusion: In our unit, neonatal sepsis caused by gram-negative organisms was highly sensitive to aminoglycosides. Almost all cases Gramnegative sepsis were adequately covered by carbapenem.

Key words: Antibiotics resistance; Bacteriological profile; Gram negative; Neonatal sepsis

\section{Introduction}

Sepsis, especially when caused by Gram-negative organisms, is a leading cause of neonatal mortality. The prevalence of neonatal Gram-negative sepsis varies among institutions and may be as high as $78 \%$ [1-4]. It is often associated with a poor neonatal outcome [5]. The causative organisms and their susceptibility to antibiotics are continuously changing over time and from one neonatal unit to another. The emergence of multidrug resistance (MDR) among these organisms is a major challenge to the medical team. The epidemiology of resistance in Gram-negative bacteria (GNB) can vary considerably by geographical locations. In a recent global surveillance report by the World Health Organization (WHO), in the Eastern Mediterranean Region, carbapenem resistance rates in Klebsiella species varied from zero to fifty four percent. Resistance to third generation cephalosporins was reported in $17-50 \%$ of isolated organisms 6. National data from the United Arab Emirates (UAE) - Abu Dhabi 2012, revealed that E.coli and Klebsiella pneumoniae resistance to third generation cephalosporins were ( $22 \%$ and $17 \%$ respectively), with a $1.5 \%$ carbapenem resistance rate [6].

Standard medical care recommends the immediate administration of empiric antibiotic therapy as soon as sepsis is suspected, ideally within one hour [7], in addition to resuscitation and stabilization. The initial choice of antibiotics is based on the likely pathogens, and the antibiotic susceptibility patterns of organisms in a NICU or data in the region. Empiric therapy for sepsis that can be caused by MDR organisms may lead to unnecessary over 
treatment with broad spectrum antibiotics or with inadequate initial antibiotics, putting these infants at higher rate of complications [8-9]. In addition, as overuse of antibiotics has been linked to the emergence of resistant organisms, it should therefore be avoided as much as possible [10]. Identifying the specific bacteriological profile and the antibiotic susceptibilities of the etiologic agents in a clinical setting can be of major help in formulating site-specific guidelines for treating sepsis and formulating effective antibiotic stewardship.

There is a paucity of data from the United Arab Emirates (UAE) on neonatal sepsis caused by Gram-negative organisms. This study aims to evaluate the prevalence of Gram-negative pathogens in neonates managed in our institution, their antibiotic susceptibility, the appropriateness of the empirical antibiotics initially prescribed, and the clinical outcomes.

\section{Methods}

This retrospective observational study includes all neonates with Gram-negative sepsis managed in the neonatal intensive care unit (NICU) at Tawam hospital over a 5-year period, from 1st January 2011 to 31st December 2015. Tawam hospital is a tertiary teaching hospital with a level III NICU which is one of the largest in the United Arab Emirates (UAE). It cares for neonates > 22 weeks of gestation and has a capacity of 41 beds equipped with modern devices. The distance between incubators is $220 \mathrm{~cm}$, handwashing units are available in each room as well as at the entrance to the unit, in addition to hand sanitizers available at the bedside. Family visiting is allowed 24 hours a day. The unit is staffed daily by three consultant neonatologists, three neonatal specialists, four residents, 18 nurses and two respiratory therapists.

The electronic medical records of all infants admitted to NICU during the study period were reviewed. The infants with Gramnegative pathogens isolated in the blood culture were identified using the NICU microbiology database. Their electronic medical records were reviewed individually, and the relevant data entered in a specifically designed data sheet. Demographic data included gestational age, birth weight, gender, mode of delivery, and the postnatal age when Gram-negative bacteraemia was recorded. Illness severity was evaluated with the Neonatal Therapeutic Intervention Scoring System (NTISS) [11-13]. Data on risk factors included mechanical ventilation, presence of a central line, and the use of total parenteral nutrition. Microbiological information included the causative organism with its antibiotic sensitivities, the initial empirical antibiotics administered when sepsis was suspected. Clinical outcomes included mortality, necrotizing enterocolitis (NEC), intraventricular hemorrhage (IVH), periventricular leukomalacia, retinopathy of prematurity (ROP) stage three or above, and bronchopulmonary dysplasia (BPD).

\section{Definitions}

Early mortality was defined as death within seven days of infection and late mortality as death occurring after seven days. Bronchopulmonary dysplasia was defined as need of supplemental oxygen or respiratory support at 36 weeks postmenstrual age. Multidrug resistance (MDR) bacteria was defined as bacteria nonsusceptible to at least one antimicrobial agent from three or more antimicrobial classes. Double antibiotic coverage for Gram-negative sepsis was defined by the administration of two of the following antibiotics (gentamicin, cephalosporins, piperacillin/tazocin, or meropenem). Adequate antibiotic coverage was defined as using empirical antibiotics to which the isolated pathogen has been shown to be sensitive.

\section{Statistical analysis}

Proportions and percentages were compared using the Chi squared test, or the Fisher's exact test for small samples when applicable. The continuous variables were reported as medians and interquartile range [IQR] as they did not follow a normal distribution (Shapiro-Wilk test). Odds ratios (OR) for outcomes were calculated with 95\% confidence intervals (ci). The STATA package version 15 (Stata Corp, College Station, Texas) was used for all analyses, and a two-tailed $\mathrm{P}$ value $<0.05$ defined statistical significance.

\section{Ethical approval}

The research was approved by the Al Ain Medical District Human Research Ethics Committee (AAMDHREC Protocol No.52117).

\section{Results}

During the study period 2732 neonates were admitted to the NICU of whom 80 infants (2.9\%) had a blood culture-proven sepsis with a Gram-negative pathogen. The prevalence ranged from (2.3\%) in 2013 to (3.8\%) in 2015. When sepsis developed, 54 out of 80 infants $(67 \%)$ were receiving total parenteral nutrition (TPN), 48 (60\%) were mechanically ventilated and 35 (31\%) had an indwelling central venous line.

Table 1 shows the demographic characteristics and the laboratory findings. The median gestational age of enrolled infants was 29 weeks, and the median birth weight 1202 grams.

Table 1: Demographic characteristics of 80 neonates with Gram-negative sepsis.

\begin{tabular}{|c|c|}
\hline Gestational Age: & $29(25,36.7)$ weeks \\
\hline Birth Weight: & $1,202(725,2287)$ grams \\
\hline \multicolumn{2}{|c|}{ Birth Weight Group: Number (\%) } \\
\hline >2500 grams & $15(18.75)$ \\
\hline $2500-1500$ grams & $14(17.5)$ \\
\hline $1499-1000$ grams & $18(22.5)$ \\
\hline$<1000$ grams & $33(41.25)$ \\
\hline Growth Assessment: Number (\%) \\
\hline AGA (Appropriate for Gestational Age & $46(57)$ \\
\hline ELBW (Extremely Low Birth Weight) & $10(12)$ \\
\hline LGA (Large for Gestational Age) & $9(11)$ \\
\hline SGA (Small for Gestational Age) & $15(19)$ \\
\hline Age When Sepsis Diagnosed: & $19.5(8.8,66.6)$ days \\
\hline
\end{tabular}




\begin{tabular}{|c|c|}
\hline \multicolumn{2}{|c|}{ Gender: Number (\%) } \\
\hline Female & $40(50 \%)$ \\
\hline Male & $37(46 \%)$ \\
\hline Ambiguous genitalia & $3(4 \%)$ \\
\hline \multicolumn{2}{|c|}{ Mode of Delivery: Number (\%) } \\
\hline Vaginal & $38(47 \%)$ \\
\hline Cesarean section & $42(53 \%)$ \\
\hline
\end{tabular}

Results are shown as median (interquartile range) unless stated otherwise.

A total of 72 out of 80 cases (90\%) received gentamycin as part of their antibiotic empiric coverage and meropenem was used empirically in four infants (5\%). Double coverage for Gramnegative sepsis was started initially in 15 neonates (18.75\%), with subsequent double antibiotic coverage in 73 (91.2\%).
Cephalosporins were used empirically as single agent in three infants $(3.7 \%$ ). The empirical antibiotic used when sepsis was suspected was adequate in 77 (97\%) infants.

Klebsiella pneumoniae was the commonest causative organism (38\% of all cases), followed by Escherichia coli (26\%), and Pseudomonas aeruginosa (11\%) (Figure 1). Only three out of 80 organisms (3.8\%) were multi-drug resistant: one Serratia marcescens, one extended-spectrum beta-lactamases (ESBL) Klebsiella pneumoniae, and one ESBL Escherichia coli. A total of 79 (98.7\%) organisms were sensitive to meropenem and 76 (95\%) to gentamicin (Figure 1). From a total of 2,732 admissions to NICU between 2011 and 2015, the mortality rate in neonates with Gramnegative sepsis was significantly higher than in those without it: 197 infants (7.2\%) died, including 33 out of 80 (41.3\%) with Gramnegative sepsis (OR 10.6, 95\% ci 6.4 to $17.4, \mathrm{P}<0.001$ ).
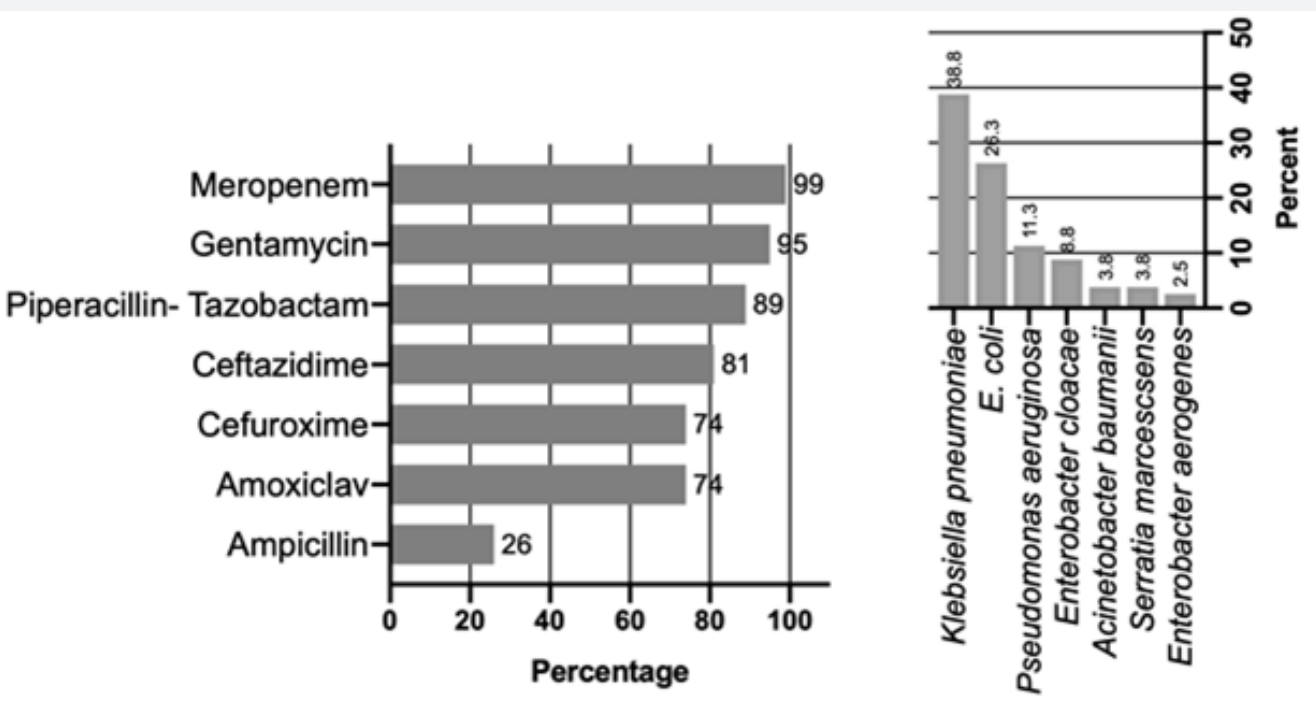

Figure 1: Distribution of pathogens identified in the blood culture of 80 neonates with Gram-negative sepsis and antibiotic sensitivities of these pathogens.

Mortality in infants with Gram-negative sepsis is shown in Table 2. Thirty-three out of 80 infants (41\%) ultimately died. Death occurred in seven out of the 23 neonates (30.4\%) who had received meropenem, compared to 26 out of 57 neonates (45.6\%) who did not receive it $(\mathrm{P}=0.316)$. The demographic characteristics between neonates who received meropenem and the others were not significantly different in term of gestational age, birth weight and the Neonatal Therapeutic Intervention Scoring System (Table 3).

Table 2: Mortality in a cohort of 80 neonates with Gram-negative sepsis.

\begin{tabular}{|c|c|}
\hline Overall mortality & 33 infants (41\%) \\
\hline Early mortality (within 7 days of the episode) & 14 infants (17\%) \\
\hline Late mortality (after 7 days of the episode) & 19 infants (24\%) \\
\hline Overall mortality for birth weight < 1500gms & 23 infants (45\%) \\
\hline $\begin{array}{c}\text { Overall mortality for birth weight 1500- } \\
\text { 2500gms }\end{array}$ & 5 infants (36\%) \\
\hline
\end{tabular}

In VLBW infants, the mortality rate and the prevalence of morbidity (NEC, BPD, IVH, PVL and ROP) was significantly higher in the presence of Gram-negative sepsis (Table 4).
Table 3: The Comparison in demographic characteristics of 80 neonates with Gram-negative sepsis who received Meropenem and who didn't.

\begin{tabular}{|c|c|c|c|}
\hline & $\begin{array}{c}\text { Received } \\
\text { Meropenem } \\
\text { (N:23) }\end{array}$ & $\begin{array}{c}\text { Didn't Receive } \\
\text { Meropenem } \\
\text { (N:57) }\end{array}$ & $\begin{array}{c}\text { P } \\
\text { Value }\end{array}$ \\
\hline Gestational age & $\begin{array}{c}28.5(25.0, \\
38.0) \text { weeks }\end{array}$ & $\begin{array}{c}29(25.5,36.4) \\
\text { weeks }\end{array}$ & $0.802^{*}$ \\
\hline Birth weight & $\begin{array}{c}1,200(720, \\
2735) \text { grams }\end{array}$ & $\begin{array}{c}1,205(730, \\
2260) \text { grams }\end{array}$ & $0.628^{*}$ \\
\hline Birth weight: number (\%) & & & $0.426 \dagger$ \\
\hline 2500 grams & $6(26.1)$ & $13(22.8)$ & \\
\hline $2500-1500$ grams & $2(8.7)$ & $8(14)$ & \\
\hline $1499-1000$ grams & $6(26.1)$ & $12(21.1)$ & \\
\hline$<1000$ grams & $9(39.1)$ & $24(42.1)$ & \\
\hline NTISS $\neq$ & $21(12,33)$ & $21(12.5,30)$ & $0.364^{*}$ \\
\hline Mortality: number $(\%)$ & $7(30.4)$ & $26(45.6)$ & $0.316 \dagger$ \\
\hline
\end{tabular}

Results are shown as median (interquartile range) unless stated otherwise.

*Kruskal Wallis test; $†$ Chi squared test or Fisher exact test for small values; $\ddagger$ The Neonatal Therapeutic Intervention Scoring System (NTISS). 
Table 4: Outcomes of Gram-negative sepsis in 51 very low birth weight infants from 468 neonates admitted from 2011 to 2015.

\begin{tabular}{|c|c|c|c|c|}
\hline & $\begin{array}{c}\text { VLBW With } \\
\text { Gram- } \\
\text { Negative } \\
\text { Sepsis (\%) }\end{array}$ & OR & $\mathbf{9 5 \% ~ c i}$ & P \\
\hline Mortality & $23(45.1)$ & 4.9 & $2.5,9.6$ & $<0.001$ \\
\hline NEC & $24(47.1)$ & 15.2 & $7.1,32.1$ & $<0.001$ \\
\hline BPD & $28(54.9)$ & 2.7 & $1.4,5.1$ & $<0.001$ \\
\hline IVH & $17(33.3)$ & 1.9 & $0.9,3.7$ & 0.038 \\
\hline PVL & $8(15.7)$ & 7.5 & $2.4,22.4$ & $<0.001$ \\
\hline ROP & $6(11.8)$ & 11.4 & $2.3,59.3$ & $<0.001$ \\
\hline
\end{tabular}

NEC: Necrotizing Enterocolitis; PVL: Periventricular Leukomalacia; BPD: Bronchopulmonary Dysplasia; IVH: Intraventricular Hemorrhage; ROP: Retinopathy of Prematurity; OR: Odds Ratio; Ci: Confidence Intervals

\section{Discussion}

The prevalence of Gram-negative sepsis was $2.9 \%$ of all neonates admitted to the NICU during the study period. This is lower than the prevalence of $4.5 \%$ reported by Mutlu et al. from Turkey [14]. The most common organisms that we have isolated were Klebsiella, E.coli and Pseudomonas aeruginosa, like a study by Kamath et al from India3. Data from five NICUs in Arab states in the Gulf region (Kuwait, Saudi Arabia, and the United Arab Emirates), reported Klebsiella was also found to be the most common organism [15].

Most Gram-negative pathogens in our study were susceptible to gentamicin. Ceftazidime and cefuroxime resistance occurred respectively in $19 \%$ and $26 \%$ of identified pathogens. Reports from the Middle East have shown a higher resistance to ceftazidime (31.5\% to $71.6 \%)$ and gentamicin (33.1\% to $43.2 \%$ ) [14-16]. The low prevalence of resistance to meropenem (1\%) in our study confirms previous reports, suggesting therefore that meropenem is a useful therapy for cases not responding to commonly used empiric therapy [14]. Al-Lawama et al have reported a high rate (28\%) of carbapenem resistance in a tertiary neonatal unit in Jordan16. Previously a study from Jordan also Khassawneh et al. [17] reported low rate to carbapenem resistance as (1.5\%). The observed differences in pathogens prevalence and their antibiotic sensitivities among the different studies may be attributed to differences in the bacterial ecosystems among the institutions, or among their clinical guidelines for antibiotic use.

The higher mortality that we have observed in infants with Gram-negative organisms compared to those without (OR 4.9, 95\% confidence intervals $2.5,9.6)$ confirm previous reports from the Canadian Neonatal Network [18]. The high mortality rate of that infection in our study (41\%), especially in very low birth weight infants (45\%), is in contrast with a lower mortality rate $(16 \%)$ reported from units in Turkey and Jordan where imipenem was used for empirical therapy [14-16]. Study from a neonatal unit in Jordan [17] where the empirical coverage for gram negative gentamicin the mortality rate was reported as (30.9\%). In our study, although not statistically significant, the mortality rate in infants who were treated with meropenem was (31\%), lower than in those who did not receive that antibiotic. One can argue that meropenem is usually added for neonates with more risk factors, or more ill-looking. The limited number of enrolled infants and the lack of randomisation did not allow us to explore this further.

Limitations of this study include its retrospective and single center design, as well as the relatively small sample size. The absence of randomisation and the lack of selected controls do not allow to draw firm conclusions on the effectiveness of the used antibiotics regarding the outcomes.

Our results demonstrate the local bacteriological ecosystem of Gram-negative neonatal sepsis in a large tertiary center in the UAE and validate our chosen initial empirical treatment.

\section{Conclusion}

Neonatal Gram-negative septicaemia has high mortality and morbidity especially in very low birth weight. Although this study was not a large randomized controlled trial, we suggest that, until larger studies involving more centers are carried out in the UAE and throughout the region, our results may serve as preliminary guidance for the antibiotic management of Gram-negative neonatal sepsis when local data remains unavailable.

\section{Acknowledgement}

None.

\section{Conflict of Interest}

We declare that there is no conflict of interest regarding the publication of this article.

\section{References}

1. Couto RC, Carvalho EA, Pedrosa TM, Pedroso ER, Neto MC, et al. (2007) A 10-year prospective surveillance of nosocomial infections in neonatal intensive care units. Am J Infect Control 35(3): 183-189.

2. Stoll BJ, Hansen N, Fanaroff AA, Wright LL, Carlo WA, et al. (2002) Lateonset sepsis in very low birth weight neonates: the experience of the NICHD Neonatal Research Network. Pediatrics 110(2 Pt 1): 285-291.

3. Kamath S, Mallaya S, Shenoy S (2010) Nosocomial infections in neonatal intensive care units: profile, risk factor assessment and antibiogram. Indian J Pediatr 77(1): 37-39.

4. Tseng YC, Chiu YC, Wang JH, Lin HC, Lin HC, et al. (2002) Nosocomial bloodstream infection in a neonatal intensive care unit of a medical center: a three-year review. J Microbiol Immunol Infect 35(3): 168-172.

5. Tsai MH, Lee IT, Chu SM, Lien R, Huang HR, et al. (2016) Clinical and Molecular Characteristics of Neonatal Extended-Spectrum $\beta$-LactamaseProducing Gram-Negative Bacteremia: A 12-Year Case-Control-Control Study of a Referral Center in Taiwan. PLoS One 11(8): e0159744.

6. World Health Organization Antimicrobial Resistance: Global Report on Surveillance.

7. Amina S Habib, Raye Linn Leukart, Amy Brown, Thomas Bartman (2018) Golden Hour for Extremely Premature Infants: Improving Time to Normothermia and Administration of IVF and Antibiotics. Pediatrics 141:1 Meeting Abstract.

8. Apisarnthanarak A, Holzmann-Pazgal G, Hamvas A, Olsen MA, Fraser VJ (2004) Antimicrobial use and the influence of inadequate empiric antimicrobial therapy on the outcomes of nosocomial bloodstream infections in a neonatal intensive care unit. Infect Control Hosp Epidemiol 25(9): 735-741.

9. Foglia EE, Fraser VJ, Elward AM (2007) Effect of nosocomial infections due to antibiotic resistant organisms on length of stay and mortality in the pediatric intensive care unit. Infect Control Hosp Epidemiol 28(3): 299-306. 
10. Centers for Disease Control and Prevention, Office of Infectious Disease Antibiotic resistance threats in the United States, 2013.

11. Gray JE, Richardson DK, McCormick MC, Workman-Daniels K, Goldmann D (1992) Neonatal Therapeutic Intervention Scoring System (NTISS) : a therapy-based severity-of-illness index. Pediatrics 90(4): 561-567.

12. Cullen DJ, Civetta JM, Briggs BA, Ferrara LC (1974) Therapeutic intervention scoring system: a method for quantitative comparison of patient care. Crit Care Med 2(2): 57-60.

13. Keene AR, Cullen DJ (1983) Therapeutic Intervention Scoring System: Update 1983. Crit Care Med 11(1): 1-3.

14. Mutlu M, Aslan Y, Saygin B, Yilmaz G, Bayramo G, et al. (2011) Neonatal Sepsis Caused by Gram-negative Bacteria in a Neonatal Intensive Care Unit: A Six Years Analysis. HK J Paediatr (new series) 16: 253-257.
15. Hammoud MS, Al-Taiar A, Al-Abdi SY, Bozaid H, Khan A, et al. (2017) Late-onset neonatal sepsis in Arab states in the Gulf region: two-year prospective study. Int J Infect Dis 55: 125-130.

16. Al-Lawama M, Badran E, Khuri-Bulos N (2014) Neonatal Gram-negative Sepsis in a Tertiary Hospital in Jordan: When Fever Means Multidrug Resistance! Al-lawama, Pediat Therapeut 4: 4.

17. Khassawneh M, Khader Y, Abuqtaish N (2009) Clinical features of neonatal sepsis caused by resistant Gram-negative bacteria. Pediatr Inter 51(3): 332-336.

18. Shah J, Jefferies AL, Yoon EW, Lee SK, Shah PS, et al. (2015) Risk Factors and Outcomes of Late-Onset Bacterial Sepsis in Preterm Neonates Born at $<32$ Weeks' Gestation. Am J Perinatol 32(7): 675-682. 